\title{
EL INNATISMO MORAL, UN NUEVO PARADIGMA DE DESARROLLO MORAL, APORTACIONES DESDE LA COGNICIÓN Y LA NEUROCIENCIA \\ THE MORAL NATIVISM, A NEW PARADIGM OF MORAL DEVELOPMENT, CONTRIBUTIONS FROM THE COGNITION AND NEUROSCIENCE
}

\author{
M. a Del Pilar Quiroga Méndez \\ Facultad de Psicología. Universidad Pontificia de Salamanca, \\ c/ Compañía 5.37002 Salamanca.quiroga@upsa.es.629879525
}

Cómo referenciar este artículo/How to reference this article:

Quiroga Méndez, M. P. (2013). El Innatismo Moral, un nuevo Paradigma de Desarrollo Moral, aportaciones desde la Cognición y la Neurociencia [The Moral Nativism, a new Paradigm of Moral Development, contributions from the Cognition and Neuroscience]. Acción Psicológica, 10(2), 179-188. http://dx.doi.org/10.5944/ap.10.2.12220

\section{Resumen}

Este trabajo presenta los nuevos abordajes en desarrollo moral, intuicionismo moral, procesamiento dual, cerebro moral, y universales morales, procedentes de la investigación generada en los últimos años. Durante más de cien años los científicos en general y la psicología en particular, han sido hostiles a la noción de que pueda existir una facultad moral o un sentido de la justicia innato En la actualidad un cuerpo importante de investigación científica procedente de la moderna ciencia cognitiva, está confluyendo en la afirmación del innatismo moral. Hoy existen evidencias suficientes para suponer que las capacidades morales básicas son no aprendidas y universales. Es razonable pensar que una especie tan intensamente social como la nuestra ha creado un modo de favorecer los procesos indispensables para la supervivencia. Por otra parte el procesamiento moral sigue una secuencia que destaca por su automaticidad, lo cuál se refrenda desde la mayor parte de las investigaciones de la psicología cognitiva y desde la neurociencia, creando todo ello un nuevo paradigma de aproximación a la comprensión del desarrollo moral.

Palabras clave: desarrollo moral, innatismo moral, cognición, neurociencia

\section{Abstract}

This paper presents new approaches to moral development, moral intuitionism, dual processing, moral brain, and universal moral; all these topics had been generated from research in recent years. For over a hundred years, scientists in general and psychology in particular have been hostile to the notion that there may be a moral faculty or an innate sense of justice Currently a significant body of scientific research from modern cognitive science, is converging in the affirmation of moral nativism. Today there is sufficient evidence to assume that the basic moral capacities are unlearned 
and universal. It is reasonable to think that a so intensely social specie like the human being, has created a way to foster the essential social and moral processes for survival. Furthermore moral processing follows a sequence that stands out for its automaticity, which is endorsed from what most of the research from cognitive psychology and from neuroscience, creating a new paradigm to approach to understanding moral development.

Keywords: moral development, moral nativism, cognition, neuroscience.

\section{Introducción}

La psicología ha desarrollado su investigación sobre la moral principalmente desde la Psicología del desarrollo, con Piaget y Kohlberg a la cabeza, pero también desde otras teorías evolutivas que la explican a través de procesos de aprendizaje, o desde procesos inconscientes. Las teorías de desarrollo moral pueden centrarse en el componente emocional de Freud, conductual de Ausubel o cognitivo de Piaget. Podemos añadir las contribuciones de la psicología de la personalidad, que interpreta el desarrollo moral como una parte del desarrollo de la identidad personal en forma de rasgo, construcción o respuesta situacional, y las aportaciones desde de la psicoterapia (Martorell, 1996) Otra parte de los estudios sobre moralidad provienen de la psicología social, con los estudios clásicos de Milgram y Zimbardo.

Aunque con evidentes diferencias de planteamiento, todas las teorías psicológicas de desarrollo moral comparten un elemento común: consideran la naturaleza básica del ser humano esencialmente negativa y socialmente construida. Los comportamientos morales son aquellos que aparecen para contrarrestar, o desplazar comportamientos asociales y egoístas. Solamente desde la perspectiva etológica y en la actualidad desde la neurociencia, se defienden posiciones alternativas a las de desconfianza esencial y construcción social, para explicar el surgimiento moral.

Darwin en el temprano 1871, insiste en que el sentido moral no es un regalo misterioso de origen desconocido, sino el resultado natural de la evolución identificable en los instintos sociales de los animales. De Waal (1996) señala que aunque existe la tendencia de ver al ser humano como esencialmente egoísta, ya en los primates se observan actitudes instintivas contrarias, que hacen más fácil la supervivencia y por tanto la preservación de la especie. En ese sentido lo moral no es una superestructura que aparece para frenar el egoísmo, sino muy al contrario, serían factores morales esenciales, los que harían al ser humano más social. No progresamos como especie a través del tiempo manteniendo comportamientos egoístas frente a la comunidad, ni a través del egocentrismo, ni por medio de la falta de empatía. Los componentes instintivos de la reciprocidad (Suchak y de Waal, 2012), la cooperación (Richerdson, Boyd y Henrich, 2003), el altruismo y la justicia, parecen tener su origen en nuestra especie social. Una especie que para sobrevivir ha de cambiar los principios de competencia y supervivencia del más apto, por características ligadas a la empatía y al altruismo de parentesco (Haidt, 2006).

Durante más de cien años los científicos en general y la psicología en particular, han sido hostiles a la noción de que pueda existir una facultad moral o un sentido de la justicia innato. En la actualidad un cuerpo importante de investigación científica, procedente de la moderna ciencia cognitiva, está confluyendo en la afirmación de la existencia de un innatismo moral, dando paso con ello a la creación de un nuevo paradigma. La disciplina emergente de la neurociencia pretende llegar a un lugar más profundo que la psicología clásica, abrir la caja negra y comprender las operaciones que suceden en ella en términos físicos (Green, 2009) Estas nuevas disciplinas, liberadas de las restricciones metodológicas paralizantes del positivismo, el conductismo, y el historicismo, han comenzado recientemente a explicar y verificar los principios innatistas del desarrollo moral (Mikhail, 2011).

\section{Intuicionismo, procesamiento dual y cerebro moral}

La investigación sobre el juicio moral en los últimos años ha configurado la existencia de una nueva ciencia de la moral con autores tan representativos como Green, Haidt, Pinker, 
Hauser, Gazzaniga, Pizarro, Nichols, Dwers, Mihkail, Bloom, Cushman, Prinz, Doris, y Carruthers. La existencia de una capacidad innata para la respuesta moral es sostenida de uno $\mathrm{u}$ otro modo por todos ellos.

La psicología moral ha estado dominada por modelos racionales que afirmaban que el conocimiento moral se alcanzaba gracias a algún tipo de razonamiento. Haidt se opone aplicando el llamado intuicionismo moral, en el que describe el sistema intuitivo y el sistema de razonamiento, como dos procesos distintos que llegan con frecuencia a diferentes juicios morales. En el primero destacan las características de rapidez, automaticidad, inaccesibilidad, procesamiento en paralelo, y dependencia del contexto. En el segundo aparecen la lentitud y el esfuerzo de procesamiento, el proceso intencional y controlado, la accesibilidad, la visibilidad, la alta demanda de recursos atencionales, la independencia del contexto, y su presencia única en la especie humana a partir de los dos años (Haidt, 2001) Ambos sistemas han sido discutidos por grandes autores como Brunner (1986) y Metcalfe y Mischel (1999).

La primacía del razonamiento moral está siendo cuestionado en la actualidad favor de la intuición moral. La mente humana está compuesta por un sistema afectivo antiguo, automático y rápido; y un sistema cognitivo filogenéticamente más nuevo, pero también más lento y motivacionalmente más débil. El razonamiento moral suele ser posterior a la decisión moral. Es después de emitir el juicio cuando el razonamiento busca y crea todos los argumentos posibles para justificar actuaciones cuyos verdaderos orígenes le son desconocidos (Haidt, 2007). Los experimentos de Gazzaniga sobre el cerebro dividido y la confabulación, explican que cuando el cerebro es informado de forma contradictoria, las personas fabrican con rapidez razones para explicar su propio comportamiento (Haidt, 2006). Así ocurre también con las sugestiones posthipnóticas, o cuando se introducen de forma subliminal palabras que interfieren en la habilidad de recordar.

El modelo del intuicionismo moral es compatible con las modernas teorías de procesa- miento dual, aunque estas no afirman la preeminencia del sistema racional en todas las ocasiones. La teoría dual explica la existencia de dos sistemas de procesamiento funcionando simultáneamente cuando se realizan juicios sobre problemas morales, de forma que es posible llegar, sobre un mismo problema, a conclusiones diferentes. El procesamiento intuitivo se activa de forma habitual, ya que es el mecanismo de resolución rápida, fácil y holística. Green mantiene que la percepción subjetiva de la moral es más importante que las verdades objetivas morales (Green, 2003; Green y Haidt, 2002) Cuando aparece algún conflicto, o cuando la situación social requiere el examen de otras facetas, es cuando aparece el razonamiento moral. Todo ello se observa en los diferentes estudios de neuroimagen y a través de la medida de los tiempos de reacción (Green, 2009) A su vez en la resolución de los dilemas personales aparece actividad en las áreas emocionales; y en los dilemas no personales surge la activación en las áreas cognitivas clásicas de razonamiento y memoria de trabajo.

Las áreas comprometidas en el juicio moral son el cortex medial prefrontal, la amígdala y la ínsula frontal. Las áreas intuitivas presionan pero no son fuerzas absolutas. Podemos tomar decisiones deliberadas en contra de esos primitivos impulsos, como probó Green con el difícil dilema del niño llorando. Una madre y su hijo están ocultos junto con otras muchas personas en un sótano, en suelo enemigo en situación de guerra, el llanto del niño alertará a los enemigos que si lo oyen matarán a todos los refugiados. En ese caso los sujetos responden lentamente y tienen una actividad incrementada en el cortex cingulado anterior, la región cerebral que responde al conflicto interno. Cuando la respuesta al dilema es: sí, mataría a mi hijo, se eleva la actividad en el cortex prefrontal dorsolateral, sugiriendo un procesamiento adicional que reemplaza la reacción inicial de horror. Existen tres formas de reemplazar respuestas intuitivas inmediatas: mediante el razonamiento consciente verbal, replanteando una misma situación desde diferentes ángulos creando un destello de intuición que compita con la primera, y hablando con otras personas para crear nuevos argumentos. 
Los hallazgos en lesionados cerebrales parecen sugerir, según observó Damasio, que hay sistemas cognitivamente disociados que contribuyen al juicio moral, existiendo una especie de módulo de moralidad o una especie de centro moral en el cerebro (Green, 2005).

\section{Los contenidos de la moral y los universales morales}

El sentido moral es un órgano de gran complejidad con rarezas que reflejan su historia evolutiva y su base neurobiológica (Pinker, 2010) El razonamiento moral es en alto grado dependiente de la estructura de la mente. Nuestras convicciones morales, lejos de ser una internalización de normas, serían más bien el regalo de nuestra propia naturaleza (Green, 2005) Pinker señala que nacemos con una gramática moral universal, que nos fuerza a analizar cada acción en términos de su estructura moral. La hipótesis de la existencia de esa gramática universal ocupa la investigación de muchos autores relevantes (Brown,1991; Cowie, 1999; Dwyer, 1999; Harman, 2008; Hauser, 2006; Mikhail, 2007, 2008, 2011; Mikhail, Sorrentino y Spelke, 1998; Pinker, 2010; Roedder, Hartman, 2010) Todos ellos buscan, basándose en el paralelismo lingüístico, la adecuación descriptiva, explicativa, conductual, neurocognitiva y evolucionista, de las cualidades morales. Para ello han de responder a las preguntas de qué y cuáles son los universales morales, cómo se adquieren, cómo se utilizan, cómo se reflejan en el cerebro, y cuál es su desarrollo evolutivo en nuestra especie.

Los universales morales se estudian a partir del dilema del tranvía, un trabajo replicado con una muestra de más de 200.000 sujetos en 120 países, obteniéndose sistemáticamente las mismas respuestas (Mikhail, 2008, 2011) Este dilema consiste en una situación en la cual un conductor de tren observa que cinco personas están en la vía, y ha de elegir si atropellarlas o cambiar el tren hacía una vía adyacente en la que se encuentra una sola persona. Nadie tiene dudas en que lo correcto es cambiar la vía y salvar a la mayoría de las personas, aunque será inevitable que una persona muera. En un segundo ejemplo se plantea la posibilidad de empujar a una persona obesa desde un puente con el objetivo de parar a un tren que va a atropellar a cinco personas. La respuesta en este caso suele ser: «No, no es moral empujar a alguien para salvar cinco vidas» Estas preguntas se responden en unos segundos, pero la cuestión para los investigadores morales es ¿por qué sacrificar una vida en el primer ejemplo es bueno, y en el segundo es malo? Y la siguiente pregunta sería ¿cómo saben las personas que hay que decir si, al primer dilema y no, al segundo? La respuesta es tan rápida que evidentemente surge de algún tipo de intuición (Green, 2005) ¿de dónde viene?, ¿por qué todo el mundo parece estar de acuerdo?

El sentido moral puede estar enraizado en el diseño del cerebro humano, y las respuestas equivalentes señalan algunos principios automáticos que aparecen rápidamente cuando se valoran las opciones en cada situación. Algunos de ellos son: la inmediatez, el contacto físico, la filiación, el daño ocasionado por acción o por omisión, el daño a un inocente, el uso del propio cuerpo, el principio del doble efecto, o la intencionalidad del daño. Green afirma que existen núcleos misteriosos de sabiduría moral aparente, que fomentan la idea de que en alguna parte del fondo de nuestra arquitectura cognitiva vamos a encontrar la gramática de un lenguaje moral innato. Gran parte del juicio moral humano depende de un mecanismo cognitivo y afectivo disociable que compite con otros que no están dedicados específicamente al juicio moral, un núcleo moral de competencia, que se organiza a través de un conjunto de reglas normativas (Green, 2005).

Pinker señala como posibles contenidos de la moral los universales que describió el antropólogo Donald E Brown: distinción entre bien y mal, empatía, justicia, admiración y generosidad, derechos y obligaciones, condena del asesinato, rapto y otras formas de violencia, sanciones, vergüenza y tabúes. Haidt (2007), cita a Shweder y a Fiske, para sugerir que existen cinco fundamentos psicológicos morales, llegando a denominarlos los colores primarios del contenido moral innato. Haidt y Joseph revisaron listas y taxonomías de valores morales propuestas por Brown (1991), Schwartz (1992) 
y Shweder, (1997) e incluyeron las aportaciones de de Waal sobre los componentes morales encontrados en primates. No crearon una taxonomía a partir de las existentes, sino que intentaron identificar cuáles eran las bases psicológicas a través de las cuales las diferentes culturas creaban la gran variedad de sistemas morales. Encontraron cinco: Daño, Justicia y equidad, Filiación y lealtad, Autoridad y respeto, Pureza y santidad (Haidt y Joseph, 2004, 2007) Estos principios se aplican a través de una serie de intuiciones cargadas emocionalmente que surgen de manera rápida y automática. Por ejemplo: acostarse con un hermano, pegar a la máxima autoridad del estado, limpiar con un trapo que es una bandera, o comerse un perro que has atropellado, son comportamientos que crean rechazo (Haidt y Kesebir, 2010).

\section{Origen y desarrollo de la intuición moral, el periodo crítico}

Quizás porque las normas morales varían en función a la cultura, la clase social y el periodo histórico, se ha pensado que la moralidad es un aprendizaje que sucede en la infancia a partir del contexto. El aprendizaje social explica la diferencia moral entre individuos procedentes de distintas culturas, épocas, o sociedades. Pero ¿qué es lo que explica las semejanzas de las respuestas morales? Y sobre todo, cómo explicar las respuestas morales que aparecen de forma automática sin apenas participación de la conciencia, o incluso en contradicción con ella.

El nuevo paradigma propone que la moralidad, igual que ocurre con el lenguaje, constituye un tipo de adaptación evolutiva construida en diferentes áreas cerebrales, propia de las especies intensamente sociales. Es una característica de especie que se explica más como capacidad emergente que aprendida, aunque por supuesto requiera del moldeamiento del contexto y la cultura. La intuición moral sería un proceso a la vez innato y derivado del medio social, desarrollado a través del proceso de externalización (Haidt y Bjorklund, 2008) dirigido por una especie de reloj biológico que indica cuándo de- ben de ponerse en funcionamiento las distintas aptitudes, siendo el ambiente un mero regulador o desencadenante del proceso (Mehler y Dupoux, 1990) Los niños por ejemplo, son indiferentes a los conceptos de justicia e igualdad hasta los cuatro años, independientemente de cuánto quieran o se esfuercen los padres en inculcar esos contenidos, y antes de los tres no comprenden el significado de las figuras de autoridad.

Existe una primacía madurativa respecto a lo propuesto por el ambiente, y solamente después de que el modelo cognitivo se ha externalizado puede ser moldeado y refinado por normas culturales que indicarán cuando y cómo deben de ser utilizadas las normas sociales. Un ejemplo de este proceso es la evolución del razonamiento frente al daño intencional versus, daño accidental. Los niños exhiben consistencia en su desarrollo en los juicios sobre daño intencional, pero existen cambios en el desarrollo respecto al juicio de daño accidental, los niños más pequeños condenan el daño accidental, mientras que los mayores y los adultos no lo hacen. Reemplazar la condena basada en resultados por exculpación del sujeto, aunque se hayan producido daños, requiere una representación enérgica del hacedor del daño (empatía), combinado con un fuerte control cognitivo. Esto explicaría que no apareciera esta posibilidad en la primera infancia sino mucho más allá, cuando la cognición se hace más fuerte y el control cognitivo más complejo (Cushman y Greene, 2011).

Evidentemente no existe función hasta que no se ha producido la maduración cognitiva suficiente para que aparezca, pero paralelamente sucede un proceso por el cual la cultura modifica, enriquece o suprime la emergencia de las intuiciones morales para crear una moralidad específica a través de la pérdida selectiva, la inmersión en las costumbres y la socialización entre pares (Haidt, 2001) La pérdida selectiva de intuiciones responde al mismo sistema de la adquisición fonológica. Nacemos con la habilidad para distinguir cientos de fonemas, pero la perdemos después de la exposición a un solo lenguaje. Una cultura que utilizara todas las potencialidades del gran arco cultural de la especie, sería tan ininteligible como un lenguaje que 
usara todos los fonemas de la lengua. Por ejemplo, un niño nace preparado para desarrollar intuiciones morales alrededor de tres categorias éticas: ética de la autonomía (protección), ética de la comunidad (familia, nación y colectividad, legalidad, modestia, autocontrol) y la ética de la divinidad (espiritualidad, piedad y pureza) (Shweder, 1997), el ambiente en el que crezca pondrá mayor énfasis en uno u otro de estos contenidos.

Existen periodos de tiempo en los que el cerebro tiene una especial plasticidad neuronal, de modo que ciertos tipos de experiencias presentadas en un tiempo determinado guiarán su cableado final. Esos periodos están bien documentados en el desarrollo de los sistemas sensoriales y en el lenguaje. El córtex frontal está siendo mielinizado desde los siete a los quince años, por lo cual puede ser este el periodo sensitivo en el cual la moralidad que proviene de la cultura es aprendida más fácilmente. (Haidt y Bjorklund, 2008) Al ser el área prefrontal la implicada mas frecuentemente en el juicio y el comportamiento moral, se sugiere que puede ser la adolescencia, mas que la infancia, el periodo crítico para el aprendizaje moral. Este extremo estaría apoyado por un interesante estudio sobre niños japoneses que habían vivido en Estados Unidos durante algún periodo por traslados laborales de sus padres Cuando los niños pasaban un tiempo en USA antes de los 9 años no se producía ningún aprendizaje emocional sobre normas y cultura, y no se derivaban efectos posteriores de esta estancia. Unos años pasado en América después de los quince años significaba un choque cultural, pero no se producían grandes cambios en la personalidad. Estos individuos conocían y podían explicar las normas americanas para el comportamiento interpersonal, la amistad, o la autopromoción, pero estas normas no estaban internalizadas Nunca se volvieron automáticas, o sentidas como evidentemente válidas, como sucede con los conocimientos adquiridos durante el periodo sensible. Este tipo de conocimientos automáticos tal como previsiblemente sucedería con el conocimiento intuitivo moral, solamente surge si se adquieren durante el periodo sensible (Minoura, 1992). Sin embargo existen algunas dificultades respecto al tiempo en el que sucede el periodo crítico y su justificación. La primera de ellas proviene de las investigaciones sobre la segunda explosión neuronal en el cerebro humano, que culminarían más allá de los 21 años (Giedd, 2008) situando su maduración en edades posteriores a la adolescencia. Por otro lado los estudios de decisión moral en niños menores de un año nos indican presencia de respuesta moral mucho antes de la maduración prefrontal (Hamlin, Wynn, y Bloom, 2007). Además no sabemos si todas las respuestas morales comparten un mismo periodo crítico de emergencia.

El desarrollo se realiza dentro de un contexto cultural en el cual el sujeto practica los comportamientos en los que está inmerso y participa de las creencias, valores, sanciones, reglas, motivos y satisfacciones asociados con ellos. Los niños aprenden sobre su cultura observando e imitando comportamientos adultos. Existe una asimetría entre cómo la cultura entra en los niños y cómo la conocen los antropólogos y experimentadores. El conocimiento cultural penetra a través de lo no verbal y la significación inconsciente, y se pretende explicar a través del lenguaje verbal, por lo cuál se pierden muchos de sus matices. Ese es un problema central de la antropología y de los estudiosos de los aspectos transculturales ligados al desarrollo moral, pues los investigadores tienen que recorrer el camino inverso: de lo verbal a lo incorporado, perdiendo información esencial en el proceso. (Haidt y Bjorklund, 2008).

\section{Primera infancia y desarrollo moral}

Sobre las emociones morales en la primera infancia destaca un trabajo sorprendente realizado con niños pequeños, en el que aparece una figura de un triángulo que intenta subir a una montaña, un cuadrado que lo impide y un círculo que ayuda. Los investigadores encontraron que los niños, de seis y de diez meses, elegían masivamente al tipo que ayudaba frente al que impedía tanto subir a la montaña, como conseguir algo guardado en una caja o jugar a la pelota. Esto sugiere que incluso a los seis meses de edad, los bebés encuentran a los que ayudan 
atractivos, y aversivos los que interfieren (Hamlin et al., 2007) Los niños, desde muy pronto, parecen hacer evaluaciones sobre las personas basadas en la naturaleza de las acciones sociales que estas llevan a cabo. Para Wynn (2008) estas evaluaciones tempranas proveen las bases necesarias para el desarrollo de un sistema de cognición moral, ya que son juicios abstractos sobre el valor de una actuación social donde el bebé no está incluido. Los seres humanos comienzan a realizar evaluaciones sociales mucho antes de lo que se había pensado, y eso supone que la capacidad para evaluar a los individuos en sus interacciones sociales es universal y no aprendida, ya que estos comportamientos comienzan a tan temprana edad que no pueden ser derivados del razonamiento superior (Haidt y Kesebir, 2010). Más bien parece que existe una especie de sistema moral perceptivo innato de muy temprana emergencia, que crea un afecto negativo hacía los que dañan y un afecto positivo hacía los que ayudan.

Sabemos que los niños llegan al mundo con un sistema de física innato que tiende a organizar el mundo circundante en dos categorías diferentes. Segun Spelke existen expectativas de los bebés frente a los objetos materiales inanimados a la temprana edad de tres meses, (continuidad espacio-temporal, solidez, cohesividad, influencia por contacto físico) Para identificar el mundo social, los bebés utilizan la atribución de intencionalidad, la morfología del rostro y los indicadores de respuesta social. Existen dos vías de procesamiento distinto para la información sobre objetos y sobre agentes sociales, los niños muy pequeños tratan a los humanos de forma diferente a otras entidades, incluso en los primeros días de vida (Spelke, 1990). Cuando una cara responsiva se hace no-responsiva los bebés se sienten perturbados, según nos indica el paradigma del rostro fijo. Sin embargo cuando un bebé de tres meses ve un objeto inanimado moviéndose, y el movimiento cesa, este pierde interés. Esto indica que los niños muy pequeños esperan que las personas y no los objetos, les respondan de manera contingente, y están muy interesados y orientados hacia el mundo social, sin que medien en estos primeros meses estructuras de pensamiento superior
Desde la Universidad de Yale, también señalan algunas sorprendentes afirmaciones sobre el desarrollo evolutivo social, coincidentes con los hallazgos de Spelke (Wynn, 2008). Existe una distinción desde los primeros meses del mundo social y el físico. Esto supone la existencia de dos sistemas cognitivos separados. La interpretación de los comportamientos del otro, objetivos, metas, y deseos, con atribuciones inherentemente mentales aparece ya a partir de los seis meses, y también surge muy tempranamente la interpretación de las acciones e interacciones en un sistema de valencias en función al impacto negativo o positivo sobre otros seres sociales. Estas tempranas evaluaciones son los análisis más precoces de nuestro mundo social, y pueden servir como la base inicial para un sistema de cognición moral que se construirá posteriormente.

Smetana (1981), por otro lado, en un estudio ya clásico demostraba que los niños desde muy temprana edad eran capaces de distinguir entre moralidad y convicción social. Niños de dos años y medio sabían que romper las normas como hablar o no guardar la ropa estaba mal, pero causar daño era mucho peor. Las normas podían cambiarse, pero pegar a otro niño, o quitarle sus cosas estaba siempre mal, independientemente del lugar donde se estuviera, e incluso aunque lo mandaran los profesores (Smetana, Killen y Turiel, 1991). El daño y saltarse las normas, parecen pertenecer a campos diferentes que los niños distinguen desde muy temprana edad. Gopnik destaca que niños de diferentes países tiene las mismas percepciones, e incluso los niños que han sufrido maltrato piensan que está intrínsecamente mal hacer daño (Gopnik, 2009) Niños de tan solo tres años responden como los adultos al dilema del tranvía (MiIkhail, 2011) A los cuatro y cinco años usan un principio de proporcionalidad para determinar el nivel de castigo correcto y muestran comprender los matices de la negligencia y la restitución (Finkel, Liss, y Moran, 1997) A los siete años ya presentan sentido de la equidad y de la justicia (Gold, Darley, Hilton y Zanna, 1984).

Estos resultados son compatibles con los que nos muestran cómo el desarrollo de la empatía se produce en los primeros meses de 
vida, y el altruismo surge mucho antes de lo que hubiéramos imaginado. Warneken y Tomasello (2006) encontraron que en niños de dieciocho, e incluso de catorce meses, son naturalmente empáticos, serviciales, y generosos y ya se observan con claridad comportamientos de ayuda sin obtención de un beneficio inmediato. Estos comportamientos son extraños desde el punto de vista evolutivo, de modo que algunos autores afirman que pertenecen únicamente a nuestra especie, pues parece improbable que todo esto se adquiera mediante instrucción explícita.

\section{Conclusiones}

Muchos comportamientos como ayudar o no dañar parecen constituir universales morales, y estar presentes desde el principio de la vida. Hoy existen evidencias suficientes para suponer que las capacidades morales son innatas y universales Es razonable pensar que una especie tan intensamente social como la nuestra ha creado un modo innato de favorecer los procesos indispensables para la supervivencia. Por otra parte el procesamiento moral sigue una secuencia que destaca por su automaticidad e innatismo, y sería fundamental conocer cuáles son los aspectos neurocognitivos a los que responde, así como su periodo crítico de instauración.

Todo ello nos sitúa en un nuevo paradigma más complejo y más respetuoso con lo esencialmente humano, que cuenta con la sabiduría innata de unos procesos que funcionan de un modo genuino, equilibrado y adaptativo. El investigador de la psicología puede observar con asombro, la aventura por la cual cada niño humano reproduce los mecanismos con los que hemos sobrevivido exitosamente durante miles de años como especie, y puede cambiar su propio modo de comprender quién es realmente ese ser humano.

\section{Referencias Bibliográficas}

Brown, D. E. (1991). Human universals. Philadelphia: Temple University Press.

Brunner, J. S. (1986). Actual minds, possible worlds. Cambridge, MA.: Harvard University Press.
Cowie, F. (1999). What's Within? Oxford: Oxford University Press.

Cushman, F. A. y Greene, J. D. (2011). Finding faults: How moral dilemmas illuminate cognitive structure. En J. Decety y J. Cacioppo (Eds.), Handbook of Social Neuroscience. New York: Oxford University Press.

De Waal, F. B. M. (1996). Good natured: The origins of right and wrong in humans and other animals. Cambridge, MA: Harvard University Press.

Dwyer, S. (1999). Moral competence. En K. Murasugi y R. Stainton (Eds.), Philosophy and Linguistics. Boulder, CO: Westview Press.

Ekman, P. (1994). All emotions are basic. En P. Ekman y R. Davidson (Eds.), The Nature of Emotion (pp. 15-19). New York: Oxford University Press.

Finkel, N. J., Liss M. B. y Moran V. R. (1997). Equal or proportional justice for accessories? Children's pearls of proportionate wisdom. Journal of Applied Development Psychology, 18(2), 229244.

Giedd, J. N. (2008). The teen brain: Insights from neuroimaging. Journal of Adolescence Health, 42(4), 335-343.

Gold, L., Darley, J. M., Hilton, J. L. y Zanna, M. P. (1984). Children's perceptions of procedural justice. Child Development, 55, 1752-1759.

Gopnik, A. (2009). The philosophical baby: what children's minds tell us about truth, love, and the meaning of life. New York: Farrar, Straus and Giroux.

Greene, J. D. (2003). From neural «is» to moral «ought»: what are the moral implications of neuroscientific moral psychology? Nature Reviews Neuroscience, 4, 847-850.

Greene, J. D. (2005). Cognitive neuroscience and the structure of the moral mind. En, S. Laurence, P. Carruthers y S. Stich (Eds.), The Innate Mind: Structure and Contents. New York: Oxford University Press.

Greene, J. D. (2009). The cognitive neuroscience of moral judgment. En M. S. Gazzaniga (Ed.), The Cognitive Neurosciences IV. Cambridge, MA.: MIT Press. 
Greene, J. D. y Haidt, J. (2002). How (and where) does moral judgment work? Trends in Cognitive Sciences, 6(12), 517-523.

Haidt, J. (2001). The emotional dog and its rational tail: A social intuitionist approach to moral judgment. Psychological Review. 108, 814-834.

Haidt, J. (2006). La hipótesis de la felicidad. Barcelona, España: Gedisa.

Haidt, J. (2007). The new synthesis in moral psychology. Science, 316, 998-1002.

Haidt, J. y Bjorklund, F. (2008). Social intuitionists answer six questions about moral psychology. En W. Sinnott-Armstrong (Ed.), Moral Psychology, Volume 2: The Cognitive Science of Morality: Intuition and Diversity (pp. 181-217). Cambridge, MA: MIT Press.

Haidt, J. y Joseph, C. (2004). Intuitive Ethics: How Innately Prepared Intuitions Generate Culturally Variable Virtues. Daedalus, pp. 55-66, Special issue on human nature.

Haidt, J., y Joseph, C. (2007). The moral mind: How 5 sets of innate moral intuitions guide the development of many culture-specific virtues, and perhaps even modules. En P. Carruthers, S. Laurence y S. Stich (Eds.), The Innate Mind, Vol. 3 (pp. 367-391). New York: Oxford.

Haidt, J. y Kesebir, S. (2010). Morality. En S. T. Fiske, D. T. Gilbert y G. Lindzey (Eds.), Handbook of Social Psychology (pp. 797. 932). New Jersey: Wiley.

Cushman, F. A., Young, L. y Hauser, M. D. (2006). The Role of Reasoning and Intuition in Moral Judgments: Testing three principles of harm. Psychological Science 17(12), 1082-1089.

Hamlin, J. K., Wynn, K. y Bloom, P. (2007). Social evaluation by preverbal infants. Nature, 450, 557-560.

Harman, G. (2000). Explaining Value and Other Essays in Moral Philosophy. New York: Oxford University Press.

Harman, G. (2008). Using a linguistic analogy to study morality. En W. Sinnott-Armstrong (Ed.), Moral Psychology, volume 1 (pp. 345-352). Londres, UK: MIT Press.

Hauser, M. (2006). Moral Minds: How Nature Designed a Universal Sense of Right and Wrong. New York: Ecco Press/Harper Collins.
Martorell J. L. (1996). Psicoterapia y desarrollo del razonamiento moral. Revista de Psicoterapia, 7(25), 17-30.

Mehler, J. y Dupoux, E. (1990). Nacer sabiendo. Introducción al desarrollo cognitivo del hombre. Madrid, España: Alianza.

Metcalfe, J. y Mischel, W. (1999). A hot/cool system analysis of delay of gratification: Dynamics of willpower. Psychological Review, 106, 3-19.

Mikhail, J. (2007). Universal moral grammar: Theory, evidence, and the future. Trends in Cognitive Sciences, 11, 143-152.

Mikhail, J. (2008). The poverty of the moral stimulus. En W. Sinnott-Armstrong (Ed.), Moral Psychology, volume 1 (pp. 353-360). Londres, UK: MIT Press.

Mikhail, J. (2011). Elements of Moral Cognition: Rawls's Linguistic Analogy and the Cognitive Science of Moral and Legal Judgment. New York: Cambridge University Press.

Mikhail, J., Sorentino, C. y Spelke, E. (1998). Toward a universal moral grammar. En Morton Ann Gernsbacher y Sharon J. Derry (Eds.), Proceedings, Twentieth Annual Conference of the Cognitive Science Society. Mahwah, New Jersey: Lawrence Erlbaum.

Minoura Y. (1992). A Sensitive Period for the Incorporation of a Cultural Meaning System: A Study of Japanese Children Growing Up in the United States. Ethos, 20, 304-339.

Pinker, S. (2010). Recuperado de http://www.nytimes.com/2008/01/13/magazine/13Psychologyt.html.

Richerson, P. J. Boyd R. y Henrich J. (2003). The Cultural Evolution of Human Cooperation. En P. Hammerstein (Ed.), The Genetic and Cultural Evolution of Cooperation (pp. 357-388). Cambridge, MA: MIT Press.

Roedder, E. y Harman, G. (2010). Linguistics and moral theory. En J. Doris (Ed.), The Moral Psychology Handbook (pp. 273-296). Oxford: Oxford University Press.

Smetana J. (1981). Preschool Children's Conceptions of Moral and Social Rules. Child Development, 52(4), 1333-1336. 
Smetana, J. G., Killen, M. y Turiel E. (1991) Children's Reasoning about Interpersonal and Moral Conflicts. Child Development, 62(3), 629-644.

Spelke, E. S. (1990). Cognitive capacities of human infants: Conceptions of object motion. En G. Edelman (Ed.), Signal and sense: Local and global order in the nervous system. NY: Wiley.

Schwartz, S. H. (1992). Universals in the content and structure of values. En M. P. Zanna (Ed.), Advances in experimental social psychology (Vol. 25, pp. 1-65). New York: Academic Press.

Shweder, R. A., Much, N. C., Mahapatra, M. y Park, L. (1997). The "big three " of morality (autonomy, community, and divinity), and the « big three » explanations of suffering. En A. Brandt y P. Rozin (Eds.), Morality and health. New York: Routledge.

Suchak, M. y de Waal, F. B. (2012). Monkeys benefit from reciprocity without the cognitive burden PNAS, 109(38), 15191-15196.

Warneken, F. y Tomasello, M. (2006). Altruistic helping in human infants and young chimpanzees. Science, 311, 1301-1303.

Wynn, K. (2008). Some innate foundations of social and moral cognition. En P. Carruthers, S. Laurence y S. Stich (Eds.), The Innate Mind: Foundations and the Future. Oxford: Oxford University Press. 